\title{
A New n-type Half-Heusler Thermoelectric Material NbCoSb
}

Lihong Huang ${ }^{\mathrm{a}, \mathrm{b}}, \mathrm{Ran} \mathrm{He}^{\mathrm{b}}$, Shuo Chen ${ }^{\mathrm{b}}$, Hao Zhang ${ }^{\mathrm{b}}$, Keshab Dahal ${ }^{\mathrm{b}}$, Haiqing Zhou ${ }^{\mathrm{b}}$, Hui Wang ${ }^{\mathrm{b}}$, Qinyong Zhang, ${ }^{\mathrm{a}, *}$ and Zhifeng Ren ${ }^{\mathrm{b}, *}$

${ }^{\mathrm{a}}$ Center for Advanced Materials and Energy, Xihua University, Chengdu, Sichuan 610039, China

${ }^{\mathrm{b}}$ Department of Physics and TcSUH, University of Houston, Houston, Texas 77204, United States

*Corresponding authors. E-mail: zren@uh.edu (Z. Ren), bohr123@163.com (Q. Zhang).

\begin{abstract}
We surprisingly made a new $n$-type thermoelectric compound $\mathrm{NbCoSb}$ with half-Heusler $(\mathrm{HH})$ structure having valence electron count of 19 , different from the traditional 18, which opens up a new route to develop new half-Heusler thermoelectric materials not following the traditional valence electron count of 18 . The samples are made by arc melting followed by ball milling and hot pressing. The effect of hot pressing temperature on the thermoelectric properties of $\mathrm{NbCoSb}$ samples has been studied. A maximum thermoelectric figure-of-merit $(Z T)$ of $\sim 0.4$ is achieved at $700{ }^{\circ} \mathrm{C}$ in $\mathrm{NbCoSb}$ sample that is hot pressed at $1000{ }^{\circ} \mathrm{C}$. This work add a new member to $\mathrm{HH}$ compounds for thermoelectric applications, although the peak $Z T$ of $\sim 0.4$ is still lower than that of the traditional HHs. Moreover, it is very interesting to see that a traditionally thought of valence electron counts of 18 is not required.
\end{abstract}

Keyword: Semiconductors; X-ray diffraction; Thermoelectrics; Electrical properties; Thermal conductivity. 


\section{Introduction}

Thermoelectric (TE) materials, which have great potentials in waste heat recovery for electric power generation and refrigeration, are experiencing a surge in research activity. ${ }^{[1,2]}$ The TE performance of a material is characterized by the dimensionless figure of merit, $Z T=\left(S^{2} \sigma / \kappa\right) \mathrm{T}$, where $S, \sigma, \kappa$, and $\mathrm{T}$ are the Seebeck coefficient, electrical conductivity, thermal conductivity, and absolute temperature, respectively. ${ }^{[3]}$ It is a great challenge to achieve high $Z T$, because the physical parameters to determine $Z T$ are interrelated and are very difficult to be manipulated separately. ${ }^{[4]}$ Lately, half-Heusler $(\mathrm{HH})$ intermetallic compounds have attracted intensive research interest because of their appealing electronic transport properties for high power factors and their robust thermal and mechanical stabilities in addition to their rich elemental combinations. ${ }^{[5-10]}$

HH compounds with the general chemical formula XYZ, where $\mathrm{X}$ can be a transition metal, a noble metal, or a rare-earth element, $\mathrm{Y}$ is a transition metal or a noble metal, and $\mathrm{Z}$ is a main group element. ${ }^{[1]} \mathrm{HH}$ compounds $\mathrm{XYZ}$ usually form the cubic MgAgAs type of structure (space group $F \overline{4} 3 \mathrm{~m}$ ). ${ }^{[12,}{ }^{13]}$ Usually good TE materials are narrow-band-gap semiconductors. Based on calculation, the basic requirement to be a TE material is that HHs compounds need to satisfy 18 valence electron count (VEC) per unit cell. Otherwise the Fermi level will enter the valence band or conduction band, and the compound will show p-type or n-type metallic properties, respectively. ${ }^{[14-18]}$ Among the HH family with 18 VEC, most research has been focused on systems like MNiSn for n-type ${ }^{[19,20]}$ and MCoSb for p-type, ${ }^{[21,22]}$ 
where $\mathrm{M}$ is individual or combination of $\mathrm{Hf}, \mathrm{Zr}$, and Ti. So far, the confirmed reported peak $Z T$ is $\sim 1.0$ at $800{ }^{\circ} \mathrm{C}$ for p-type $\mathrm{Hf}_{0.44} \mathrm{Zr}_{0.44} \mathrm{Ti}_{0.12} \mathrm{CoSb}_{0.8} \mathrm{Sn}_{0.2}{ }^{[22]}$ and $\sim 1.0$ at $500{ }^{\circ} \mathrm{C}$ for $\mathrm{n}$-type $\mathrm{Hf}_{0.5} \mathrm{Zr}_{0.25} \mathrm{Ti}_{0.25} \mathrm{NiSn}_{0.99} \mathrm{Sb}_{0.01}{ }^{[19]}$ Both of them have 18 valence electron count.

Besides $\mathrm{MNiSn}$ and $\mathrm{MCoSb}(\mathrm{M}=\mathrm{Hf}, \mathrm{Zr}$, Ti), other $\mathrm{HH}$ thermoelectric materials with 18 VEC such as NbCoSn $(Z T=0.3$ at $850 \mathrm{~K}$ for $\left.\mathrm{Nb}_{0.99} \mathrm{Ti}_{0.01} \mathrm{CoSn}_{0.9} \mathrm{Sb}_{0.1}\right),{ }^{[23,24]} \mathrm{VFeSb}(Z T=0.25 \text { at } 550 \mathrm{~K} \text { for pure } \mathrm{VFeSb})^{[25,26]}$ and so on, were also investigated in recent years. However, there is very little work on forming $\mathrm{HH}$ phase material using elements with more or less than 18 VEC for TE application. Tobola et al. ${ }^{[27]}$ calculated the electronic phase structure of many HHs using the Korringa-Kohn-Rostoker computation, and thought those with VEC $=19$ such as VCoSb, NbCoSb, and TiNiSb were metallic phases, not suitable for semiconductor or thermoelectric application. In 2008, Jiong et al. ${ }^{[17]}$ performed a theoretical evaluation of the thermoelectric-related electronic transport properties of $36 \mathrm{HH}$ compounds with VEC of 17,18 or 19 , such as TiCoSn, TiCoSb, VCoSb. However up to now, there is no experimental results about HH compounds with VEC $\neq 18$ as TE materials to verify the validity of the calculations. ${ }^{[27-29]}$ Therefore, it is scientifically very interesting to see whether a $\mathrm{HH}$ compound with VEC $\neq 18$ will show the thermoelectric performance regardless the ZT value. To our great surprise, not only we synthesized a $\mathrm{HH}$ phase $\mathrm{NbCoSb}$ that has $19 \mathrm{VEC}$, but also it is n-type with a fairly good $\mathrm{ZT}$ value, meaning $\mathrm{Nb}$ is a very strong electron donor in comparison with the p-type $\mathrm{MCoSb}$ where $\mathrm{M}$ is $\mathrm{Hf}, \mathrm{Zr}$, or $\mathrm{Ti}$, which opens a new 
route to develop new half-Heusler thermoelectric materials not following the traditional VEC of 18. In this paper, we report our surprising findings on forming $\mathrm{HH}$ phase and the TE performance of NbCoSb. We have achieved a peak $Z T$ of $\sim 0.4$ at $700{ }^{\circ} \mathrm{C}$ without any doping. It is worth pointing out that the peak ZTs for TiCoSb, $\mathrm{ZrCoSb}$, and $\mathrm{HfCoSb}$ are all less than $0.03 .{ }^{[30]}$ Although for now, the peak $Z T$ of $\mathrm{NbCoSb}$ is not high enough to be practical, it should be high enough to attract more attention to this kind of traditionally thought impossible compounds hoping to find new similar materials with much higher ZTs.

\section{Experimental Details}

Ingots of $\mathrm{NbCoSb}$ compounds were prepared by arc melting stoichiometric amounts of elements ( $\mathrm{Nb}$ slug, Co slug, Sb broken rod, $>99.8 \%$ purity, Alfa Aesar) under Ar protection for five times to ensure homogeneous alloying. An excess amount of 5\% Sb were added to compensate for the weight loss due to its high vapor pressure during arc melting. Subsequently, the alloyed ingots were ball milled (SPEX 8000M Mixer/Mill) for 7-11 $\mathrm{h}$ in a stainless steel jar to produce nanoparticles. After that, about $2.2 \mathrm{~g}$ of ball milled powders were loaded to a graphite die and pressed by a DC assisted hot pressing method into pellets with a diameter of $12.7 \mathrm{~mm}$ and thickness of $2 \mathrm{~mm}$. The hot press temperatures (HPT) were 900, 950, 1000, 1050, and $1100{ }^{\circ} \mathrm{C}$ at a pressure of $77 \mathrm{MPa}$ for $2 \mathrm{~min}$. After being naturally cooled down to room temperature, the samples were polished for the room temperature mass density measurement via Archimedes method. Individual thermoelectric property measurements were carried 
out from room temperature to $700{ }^{\circ} \mathrm{C}$. First, the thermal diffusivities $(D)$ of the pellets were measured by laser flash (LFA457, Netzch), then the samples were cut to approximately $2 \mathrm{~mm} \times 2 \mathrm{~mm} \times 12 \mathrm{~mm}$ bars for electrical conductivity $(\sigma)$ and Seebeck coefficient $(S)$ measurements (ZEM-3, ULVAC). Due to the randomness of the small grains, the samples do not show any anisotropy on properties. The rest of the samples were cut into discs and polished to less than $6 \mathrm{~mm}$ in diameter and about $1 \mathrm{~mm}$ in thickness for specific heat $(C p)$ measurements (DSC $404 \mathrm{C}$, Netzch). Hall measurements were performed at room temperature by Physical Property Measurement System (Quantum Design, PPMS with TTO) using a four-probe configuration, with sample dimensions of $4 \mathrm{~mm} \times 4 \mathrm{~mm} \times 0.5 \mathrm{~mm}$. Then the carrier concentration $n$ and Hall mobility $\mu_{H}$ were calculated using $n=-\left(1 / q R_{H}\right)$ and $\mu_{H}=\sigma R_{H}$, respectively, where $R_{H}$ is the Hall coefficient. The hot pressed dense bulk samples were characterized by X-ray diffraction (Panalytical X'pert), scanning electron microscope (SEM, LEO 1525), and high-resolution transmission electron microscope (HRTEM, JEOL 2100F) to study their crystallinity, phase formation, average grain size, and grain size distribution.

\section{Results and Discussion}

All of our samples are prepared by the same process: arc melting, ball milling, hot pressing, and then polishing. Figure 1 shows the X-ray diffraction (XRD) patterns of $\mathrm{NbCoSb}$ samples hot pressed at different temperatures. All diffraction peaks are well matched with the cubic HH phase of NbCoSb (JCPDS 51-1247, $a=0.5897 \mathrm{~nm}$ ). Very 
weak diffraction peaks for $\mathrm{Nb}_{3} \mathrm{Sb}$ are also identified, which is resulted from the peritectic reaction during the arc-melting process. As shown in Table 1, the lattice constants of our hot-pressed samples decrease from $0.5901 \mathrm{~nm}$ for the sample hot pressed at $900{ }^{\circ} \mathrm{C}$ to $0.5896 \mathrm{~nm}$ for that hot pressed at $1100{ }^{\circ} \mathrm{C}$. The slight decreasing trend might be related to a very minor composition difference due to possibly different amount of impurity phase caused by the hot pressing temperature. The theoretical density of the samples can be calculated by $\rho_{\text {cal }}=\sum n_{\mathrm{i}} \mathrm{M}_{\mathrm{i}} /\left(a^{3} N_{A}\right)$, where $n_{\mathrm{i}}$ is the number of atoms per unit cell of $\mathrm{Nb}, \mathrm{Co}$, and $\mathrm{Sb}, \mathrm{M}_{\mathrm{i}}$ is the corresponding atomic mass of each element, $a$ is the lattice constant, and $N_{A}$ is the Avogadro constant (6.023 $\times 10^{23} \mathrm{~mol}^{-1}$ ). The calculated theoretical densities are listed in Table 1 together with the measured values and relative percentages. All samples with hot press temperature (HPT) being equal or greater than $1000{ }^{\circ} \mathrm{C}$ show densities over $94 \%$ of their theoretical densities, while samples with HPT $<1000{ }^{\circ} \mathrm{C}$ show densities about $88 \sim 89 \%$ of their theoretical densities.

Figure 2 shows the typical scanning electron microscopic (SEM) images on the freshly fractured surfaces of the $\mathrm{NbCoSb}$ samples hot pressed at different temperatures. SEM images of the samples HPT900 and PT950, hot pressed at 900 and $950{ }^{\circ} \mathrm{C}$, respectively, are shown in Figure $2 \mathrm{a}$, b. Due to the lower hot press temperature, there are clearly holes, which is consistent with their lower density values. Figure $2 \mathrm{c}-\mathrm{f}$ show that all the hot pressed samples with HPT $\geq 1000{ }^{\circ} \mathrm{C}$ look like densely packed (Fig. $2 \mathrm{~d}$ is the enlarged view of 2c). However the density measurement shows a relative density of only about $94 \%$, which is probably due to 
the loss of $\mathrm{Sb}$ that caused the theoretical density lower than calculated values shown in Table 1. The effects of hot pressing temperature on the grain size and distribution are clearly shown in the SEM images. Figure $2 \mathrm{a}-\mathrm{f}$ show that the average grain size is about $\sim 100, \sim 200, \sim 400, \sim 700$, and $\sim 1000 \mathrm{~nm}$ for the samples hot pressed at 900, 950, 1000,1050 , and $1100{ }^{\circ} \mathrm{C}$, respectively. The particle size is not uniform when HPT is higher than $1050{ }^{\circ} \mathrm{C}$, some grains are smaller, with a diameter of several hundred nanometers, while some are bigger, with a diameter of about one micrometer (Figure 2e, f).

Transmission electron microscope (TEM) study of the NbCoSb samples hot pressed at $1000{ }^{\circ} \mathrm{C}$ has been performed. First, cutting the bulk sample into appropriate size (generally less than $3 \times 2.5 \mathrm{~mm}$ ), then polishing it until the thickness is about $50 \mu \mathrm{m}$, finally the sample was thinned to $10 \mathrm{~nm}$ by ion milling. Figure 3a clearly shows that the sample is densely packed polycrystalline and contains grains of around 200-400 nm, which is consistent with the SEM study (Figure 2c, d). Figure $3 b$ shows a high-resolution TEM (HRTEM) image of the lattice fringes and grain boundaries between three adjacent grains. It clearly indicates that individual grains are highly crystalline and the grain boundaries are clean. Since the polycrystalline samples are prepared by hot pressing the ball milled powders, the grain orientation of the adjacent grains are random, which promotes phonon scattering.

The thermal diffusivities are plotted in Figure 4a. It is clear that the thermal diffusivity is higher when the hot press temperature is higher because of the larger 
grain size and fewer defects. Specific heat capacity $(C p)$ is also measured, as plotted in Figure $4 \mathrm{~b}$. Among all the samples, the sample hot pressed at $1000{ }^{\circ} \mathrm{C}$ shows the lowest $C p$. Normally $C p$ should not change if the composition is fixed. Here we have a little bit difference in $C p$ s for samples hot pressed at different temperatures, which is the result of different compositions resulted from $\mathrm{Sb}$ loss at the high hot pressing temperatures. When $\mathrm{Sb}$ is lost during hot pressing, the $C p$ should increase since $\mathrm{Sb}$ has the lowest $C p$ among all the three elements. The thermal conductivity is then calculated by multiplying the density, specific heat capacity, and thermal diffusivity of each sample. As shown in Figure 4c, the total thermal conductivity increases with increasing HPT. We further tried to estimate the lattice thermal conductivity from the relationship: $\kappa=\kappa_{L}+\kappa_{e}+\kappa_{b i}$. Here, the electronic thermal conductivity $\left(\kappa_{e}\right)$ is calculated according to the Wiedemann-Franz relation, ${ }^{[31]} \kappa_{e}=L \sigma \mathrm{T}$, where $L$ is the Lorenz number and $\mathrm{T}$ the absolute temperature. To calculate the Lorenz number, we used the electrical conductivity and Seebeck coefficient shown in Figure 5a, b. The Lorenz number is calculated from the reduced Fermi energy, which is deduced from the Seebeck coefficient and based on a single-band model for all samples. ${ }^{[32,33]}$ The Lorenz number is found to be $2.24 \times 10^{-8} \mathrm{~W} \Omega \mathrm{K}^{-2}$ at room temperature, which decreases to $1.81 \times 10^{-8} \mathrm{~W} \Omega \mathrm{K}^{-2}$ at $700{ }^{\circ} \mathrm{C}$ for sample HPT1000. Then, the calculated electronic thermal conductivity is subtracted from the measured total thermal conductivity to obtain the lattice thermal conductivity, and the bipolar thermal conductivity is assumed to be negligibly small because the temperature dependent Seebeck coefficient is monotonically increasing. As shown in Figure 4d, the lattice 
thermal conductivity increases with increasing HPT. The lowest lattice thermal conductivity in sample HPT900 is related to the significant phonon scattering effect at grain boundaries owing to its smallest grain size and pores in the sample. ${ }^{[34-36]}$

The temperature dependence of the electrical conductivity $\sigma$, Seebeck coefficient $S$, power factor $S^{2} \sigma$, and $Z T$ of our hot pressed NbCoSb samples are plotted in Figure 5. All samples show metallic behavior as the electrical conductivity decreases with increasing temperature, which indicates a stronger electron scattering at higher temperatures (Figure 5a). It is generally observed that increasing HPT produces higher electrical conductivity, but the increasing of electrical conductivity by higher HPT gradually diminishes after HPT is higher than $1000{ }^{\circ} \mathrm{C}$. Figure $5 \mathrm{~b}$ shows that the Seebeck coefficient decreases with increasing hot pressing temperature and increase with increasing the measuring temperature over the entire temperature range of $25-700{ }^{\circ} \mathrm{C}$, which follows the normal trend that Seebeck coefficient is always inversely related to the electrical conductivity presented in Figure 5a. All measured Seebeck coefficient are negative, indicating $n$-type transport behavior, which is a big surprising to us since $\mathrm{MCoSb}$ are all strong p-types when $\mathrm{M}$ is $\mathrm{Ti}, \mathrm{Zr}$, and $\mathrm{Hf}$. It seems $\mathrm{Nb}$ is a very strong electron donor. Another feature we noted is that the temperature dependent Seebeck shows almost a linear increase up to $700{ }^{\circ} \mathrm{C}$, suggesting no bipolar effect in our samples before $700{ }^{\circ} \mathrm{C}$. The maximum is not reached even at $700{ }^{\circ} \mathrm{C}$, which makes $\mathrm{NbCoSb}$ possible for even higher temperature applications. Consequently, the power factor is calculated and presented in Figure 5c. The maximum power factor is $21.6 \mu \mathrm{W} \mathrm{cm} \mathrm{cm}^{-1}$ at $700{ }^{\circ} \mathrm{C}$ for sample HPT1000. The 
power factor is about half of that of other good $\mathrm{HH}$ materials. ${ }^{[20]}$

The Hall voltage $V_{H}$ at room temperature is measured by the Physical Property Measurement System. The carrier concentration $n$, Hall coefficient $R_{H}$, and carrier mobility $\mu_{H}$ are calculated by the formula $n=\mathrm{IB} / V_{H} q \mathrm{t}, R_{H}=-(1 / q n), \mu_{H}=\sigma R_{H}$, respectively, where $\mathrm{I}$ is the current $(8 \mathrm{~mA}), \mathrm{B}$ is the magnetic field intensity $(3 \mathrm{~T}), q=$ $-e=1.602 \times 10^{-9} \mathrm{C}, \mathrm{t}$ is the thickness of sample, $\sigma$ is the electrical conductivity obtained from ZEM measurement before. Table 2 summarizes the transport properties of $\mathrm{NbCoSb}$ at room temperature. Hall coefficients have negative values for all the samples, indicating $n$-type conduction with electrons as major carriers, in agreement with the Seebeck measurement by transport method using ZEM-3. It can be observed that the resistivity decreases with increasing the hot-pressing temperature up to $1000{ }^{\circ} \mathrm{C}$ and then increases. It is mainly because the variation of mobility that increased with the hot-pressing temperature up to $1000{ }^{\circ} \mathrm{C}$ and then decreased.

By combining the electrical properties with thermal conductivity, we obtained a maximal power factor of about $21.6 \mu \mathrm{W} \mathrm{cm} \mathrm{c}^{-1}$ and $Z T$ of approximately 0.4 at $700{ }^{\circ} \mathrm{C}$ for the sample HPT1000 (Figure 5d). Clearly, the best hot pressing temperature is between 950 and $1000{ }^{\circ} \mathrm{C}$. When it is too low or high, the $Z T$ is lower due to lower density or larger grains, respectively.

Surprisingly, we have successfully made a new n-type half-Heusler compound $\mathrm{NbCoSb}$ with the valence electron count of 19 , different from the traditionally thought of 18, which opens up a new route to develop new half-Heusler thermoelectric 
materials not following the VEC of 18 . Comparing with a typical $n$-type $\mathrm{HH}$ $\mathrm{Hf}_{0.15} \mathrm{Zr}_{0.85} \mathrm{NiSn}_{0.99} \mathrm{Sb}_{0.01}{ }^{[20]}$ we reported before, $\mathrm{NbCoSb}$ has higher electrical conductivity, lower Seebeck coefficient and almost the same thermal conductivity. Even though the peak $Z T$ of $\sim 0.4$ is still too low, it is worth pointing out that all the traditional $\mathrm{HH}$ materials without any doping such as TiCoSb, $\mathrm{ZrCoSb}$, and $\mathrm{HfCoSb}$ have peak $Z T s$ only about 0.03 , which is much smaller than 0.4 . Therefore, it is very interesting to see that a traditionally thought of valence electron counts of 18 is not required. And we believe that it is very hopeful to further improve the thermoelectric properties of $\mathrm{NbCoSb}$ with proper doping. More promising is that the working temperature can be even higher than $700{ }^{\circ} \mathrm{C}$ for $\mathrm{NbCoSb} \mathrm{HH}$ compound.

\section{Conclusions}

In conclusion, we have succeeded in preparing a new n-type half-Heusler compound $\mathrm{NbCoSb}$ with the valence electron count of 19 , via arc-melting, ball milling, and direct current assisted hot pressing. We found that the hot press temperature plays an important role in the thermoelectric properties of $\mathrm{NbCoSb}$. A maximum peak $Z T$ of $\sim 0.4$ is observed at $700{ }^{\circ} \mathrm{C}$ for $\mathrm{NbCoSb}$ samples hot pressed at both 950 and $1000{ }^{\circ} \mathrm{C}$. Even though the peak $Z T$ of $\sim 0.4$ is still too low for practical applications, it is very interesting to see that a traditionally thought of valence electron counts of 18 is not required, which points out that many new $\mathrm{HH}$ compounds may be synthesized for possible medium to high temperature applications. 


\section{Acknowledgements}

The work performed at Xihua University is supported by the Research Project of

Education Department of Sichuan Province (No. 14ZB0133), Natural Science Foundation of China (No. 51372208) and that at the University of Houston is supported by "Solid State Solar Thermal Energy Conversion Center ( ${ }^{3}$ TEC)", an Energy Frontier Research Center funded by the U.S. Department of Energy, Office of Science, Office of Basic Energy Science under award number DE-SC0001299. 


\section{References}

[1] G.J. Snyder, E.S. Toberer, Nat. Mater. 7 (2008) 105-114.

[2] M.S. Dresselhaus, G. Chen, M.Y. Tang, R.G. Yang, H. Lee, D.Z. Wang, Z.F. Ren, J.P. Fleurial, P. Gogna, Adv. Mater. 19 (2007) 1043-1053.

[3] H.J. Goldsmid, in CRC Handbook of Thermoelectrics, (Eds: D. M. Rowe), CRC Press, Boca Raton, FL, 1995, Ch. 3.

[4] A. Shakouri, Annu. Rev. Mater. Res. 41 (2011) 399-431.

[5] X. Yan, G. Joshi, W. Liu, Y. Lan, H. Wang, S. Lee, J.W. Simonson, S.J. Poon, T.M. Tritt, G. Chen, Z.F. Ren, Nano Lett. 11 (2011) 556-560.

[6] X. Yan, W. Liu, H. Wang, S. Chen, J. Shiomi, K. Esfarjani, H. Wang, D. Wang, G. Chen, Z.F. Ren, Energy Environ. Sci. 5 (2012) 7543-7548.

[7] K. Bartholome, B. Balke, D. Zuckermann, M. Köhne, M. Müller, K. Tarantik, J. König, J. Electron. Mater. 43 (2014) 1775-1781.

[8] P. Maji, J.P.A. Makongo, X. Zhou, H. Chi, C. Uher and P.F.P. Poudeu, J. Solid State Chem. 202 (2013) 70-76.

[9] T. Graf, C. Felser, S.S.P. Parkin, Prog. Solid State Ch. 39 (2011) 1-50.

[10] M. Zebarjadi, K. Esfarjani, M.S. Dresselhaus, Z.F. Ren, G. Chen, Energy Environ. Sci. 5 (2012) 5147-5162.

[11] K. Momma, F. Izumi, J. Appl. Cryst. 41 (2008) 653-658.

[12] S.H. Wang, H.M. Cheng, R.J. Wu, W.H. Chao, Thin Solid Films 518 (2010) $5901-5904$.

[13] Y. Kawaharada, K. Kurosaki, H. Muta, M. Uno, S. Yamanaka, J Alloys Compd. 
377 (2004) 312-315.

[14] W.S. Liu, X. Yan, G. Chen, Z.F. Ren, Nano Energy 1 (2012) 42-56.

[15] C.S. Birkel, W.G. Zeier, J.E. Douglas, B.R. Lettiere, C.E. Mills, G. Seward, A. Birkel, M.L. Snedaker, Y.C. Zhang, G.J. Snyder, T.M. Pollock, R. Seshadri, G.D. Stucky, Chem. Mater. 24 (2012) 2558-2565.

[16] G. Joshi, X. Yan, H.Z. Wang, W.S. Liu, G. Chen, Z.F. Ren, Adv. Energy Mater. $1(2011)$ 643-647.

[17] J. Yang, H.M. Li, T. Wu, W.Q. Zhang, L.D. Chen, J.H. Yang, Adv. Funct. Mater. 18 (2008) 2880-2888.

[18] C. Yu, T.J. Zhu, R.Z. Shi, Y. Zhang, X.B. Zhao, J. He, Acta Mater. 57 (2009) $2757-2764$.

[19] G. Joshi, T. Dahala, S. Chen, H. Wang, J. Shiomib, G. Chen, Z. Ren, Nano Energy 2 (2013) 82-87.

[20] S. Chen, K.C. Lukas, W. Liu, C.P. Opeil, G. Chen, Z.F. Ren, Adv. Energy Mater. 3 (2013) 1210-1214.

[21] S.J. Poon, D. Wu, S. Zhu, W.J. Xie, T.M. Tritt, P. Thomas, R. Venkatasubramanian, J. Mater. Res. 26 (2011) 2795-2802.

[22] X. Yan, W.S. Liu, S. Chen, H. Wang, Q. Zhang, G. Chen, Z.F. Ren, Adv. Energy Mater. 3 (2013) 1195-1200.

[23] Y. Ono, S. Inayama, H. Adachi, T. Kajitani, Jap. J. Appl. Phys. 45 (2006) 8740-8743.

[24] M.A. Kouacou, A.A. Koua, J.T. Zoueu, K. Konan, J. Pierre, Pramana-J. Phys. 
71 (2008) 157-166.

[25] M.M. Zou, J.F. Li, P.J. Guo, T. Kita, J. Solid State Chem. 198 (2013) 125-130.

[26] C.G. Fu, H.H. Xie, Y.T. Liu, T.J. Zhu, J. Xie, X.B. Zhao, Intermetallics 32 (2013) 39-43.

[27] J. Tobola, J. Pierre, J. Alloy. Compd. 296 (2000) 243-252.

[28] C.B.H. Evers, C.G. Richter, K. Hartjes, W. Jeitschko, J. Alloy. Compd. 252 (1997) 93-97.

[29] G. Melnyk, E. Bauer, P. Rogl, R. Skolozdra and E. Seidl, J. Alloy. Compd. 296 (2000) 235-242.

[30] T. Sekimoto, K. Kurosaki, H. Muta and S. Yamanaka, Mater. Trans. 46 (2005) $1481-1484$

[31] N.W. Ashcroft, D.N. Mermin, Solid State Physics, Brooks Cole, Stamford, CT, 1976.

[32] D.M. Rowe, C.M. Bhandari, Modern thermoelectric, Reston Publishing Company, Reston, VA, 1983.

[33] W.S. Liu, Q.Y. Zhang, Y.C. Lan, S. Chen, X. Yan, Q. Zhang, H. Wang, D.Z. Wang, G. Chen, Z.F. Ren, Adv. Energy Mater. 1 (2011) 577-587.

[34] C. Yu, H. Xie, C. Fu, T. Zhu, X. Zhao, J. Mater. Res. 27 (2012) 2457-2465.

[35] G.J. Snyder, E.S. Toberer, Nat. Mater. 7 (2008) 105-114.

[36] J.Q. He, J.R. Sootsman, S.N. Girard, J.C. Zheng, J.G. Wen, Y.M. Zhu, M.G. Kanatzidis, V.P. Dravid, J. Am. Chem. Soc. 132 (2010) 8669-8675. 
Table 1. Lattice constant, theoretical, experimental, and relative density of $\mathrm{NbCoSb}$ samples hot pressed at different temperatures.

\begin{tabular}{cccccc}
\hline Sample & Hot-press & Lattice & Theoretical & Experimental & Relative \\
name & $\begin{array}{c}\text { demperature } \\
\left({ }^{\circ} \mathrm{C}\right)\end{array}$ & $\begin{array}{c}\text { constant } \\
(\mathrm{nm})\end{array}$ & $\begin{array}{c}\text { density } \\
\left(\mathrm{g} \mathrm{cm}^{-3}\right)\end{array}$ & $\begin{array}{c}\text { density } \\
\left(\mathrm{g} \mathrm{cm}^{-3}\right)\end{array}$ & $(\%)$ \\
\hline HP900 & 900 & 0.5901 & 8.844 & 7.805 & 88.25 \\
HP950 & 950 & 0.5900 & 8.848 & 7.931 & 89.64 \\
HP1000 & 1000 & 0.5899 & 8.853 & 8.329 & 94.08 \\
HP1050 & 1050 & 0.5898 & 8.857 & 8.332 & 94.07 \\
HP1100 & 1100 & 0.5896 & 8.866 & 8.376 & 94.47 \\
\hline
\end{tabular}

Table 2. Resistivity, carrier concentration, and mobility at room temperature of $\mathrm{NbCoSb}$ samples hot pressed at different temperatures.

\begin{tabular}{cccccc}
\hline Sample & HPT & Hall coefficient & Resistivity & Carrier concentration & Mobility \\
name & $\left({ }^{\circ} \mathrm{C}\right)$ & $\left(10^{-9} \mathrm{~m}^{3} \mathrm{C}^{-1}\right)$ & $\left(10^{-6} \Omega \mathrm{m}\right)$ & $\left(10^{21} \mathrm{~cm}^{-3}\right)$ & $\left(\mathrm{cm}^{2} \mathrm{~V}^{-1} \mathrm{~s}^{-1}\right)$ \\
\hline HP900 & 900 & -1.14 & 7.84 & 5.48 & 1.45 \\
HP950 & 950 & -0.83 & 4.16 & 7.49 & 2.01 \\
HP1000 & 1000 & -1.03 & 2.85 & 6.04 & 3.62 \\
HP1050 & 1050 & -1.01 & 2.92 & 6.21 & 3.45 \\
HP1100 & 1100 & -1.02 & 3.11 & 6.09 & 3.30 \\
\hline
\end{tabular}




\section{Figure captions}

Figure 1. X-ray diffraction patterns of $\mathrm{NbCoSb}$ samples hot pressed at different temperatures.

Figure 2. SEM images of $\mathrm{NbCoSb}$ samples hot pressed at different temperatures.
(a) $900{ }^{\circ} \mathrm{C}$
${ }^{\circ} \mathrm{C}$, (b) $950{ }^{\circ} \mathrm{C}$
${ }^{\circ} \mathrm{C},(\mathrm{c}, \mathrm{d}) 1000^{\circ} \mathrm{C}$
${ }^{\circ} \mathrm{C},(\mathrm{e})$
$1050{ }^{\circ} \mathrm{C}$, and (f) $1100{ }^{\circ} \mathrm{C}$.

Figure 3. Medium (a) and high (b) resolution TEM images of NbCoSb samples hot pressed at $1000{ }^{\circ} \mathrm{C}$.

Figure 4. Temperature dependent thermal properties of NbCoSb samples hot pressed at different temperatures. (a) Thermal diffusivity, (b) specific heat capacity, (c) thermal conductivity, and (d) lattice thermal conductivity.

Figure 5. Temperature dependent thermoelectric properties of $\mathrm{NbCoSb}$ samples hot pressed at different temperatures. (a) Electrical conductivity, (b) Seebeck coefficient, (c) power factor, and (d) $Z T$ value. 


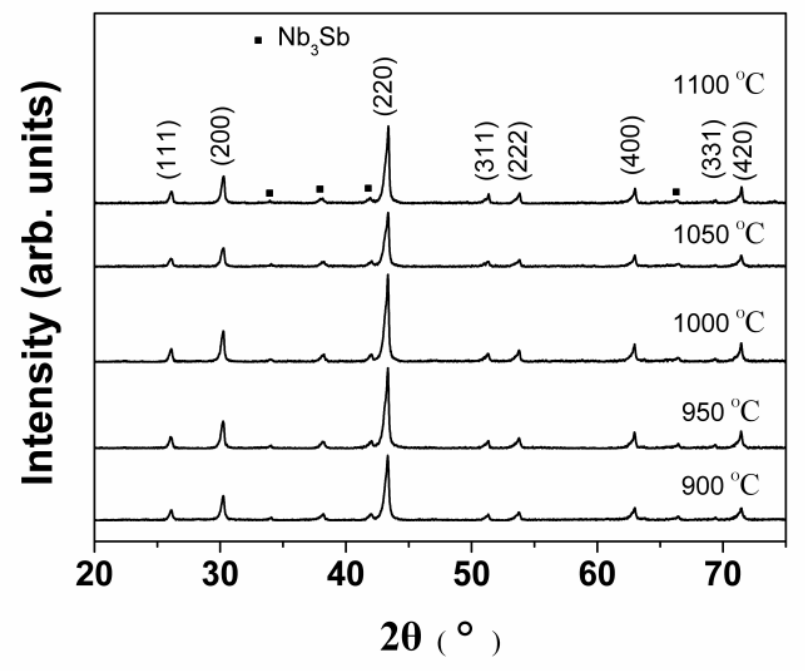

Figure 1 


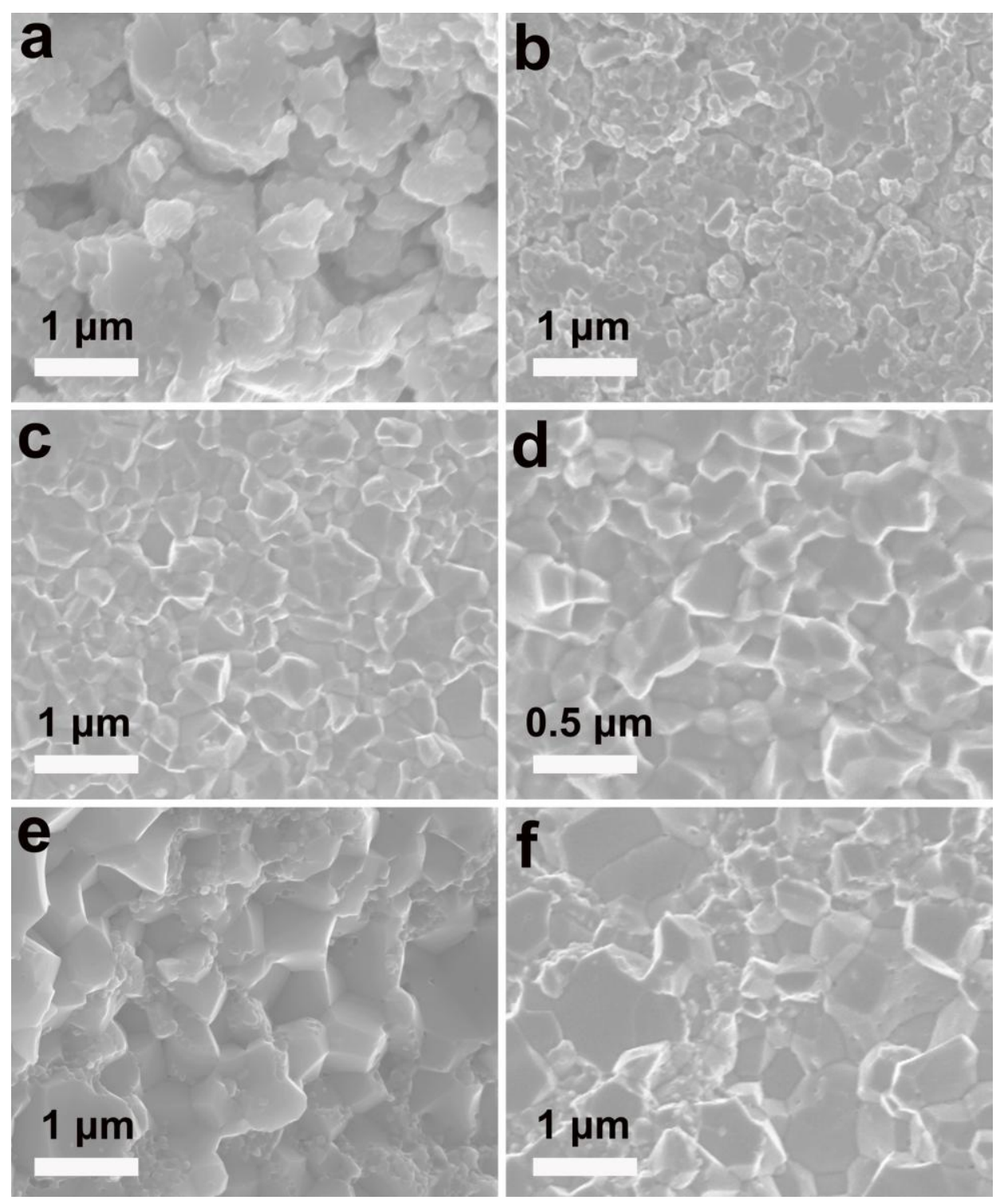

Figure 2 


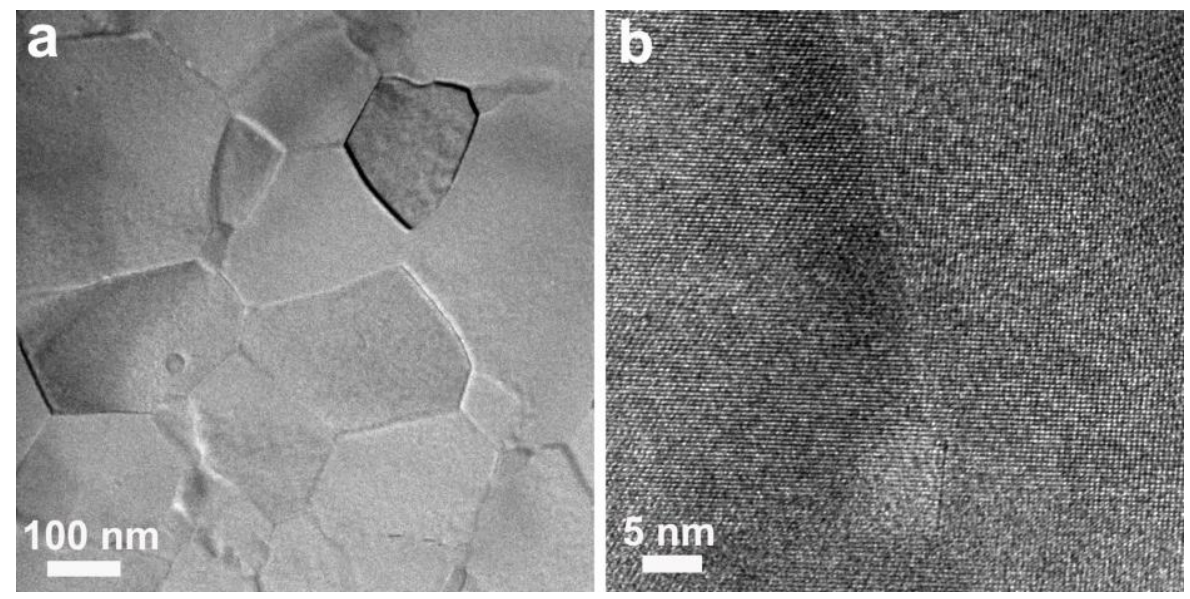

Figure 3 

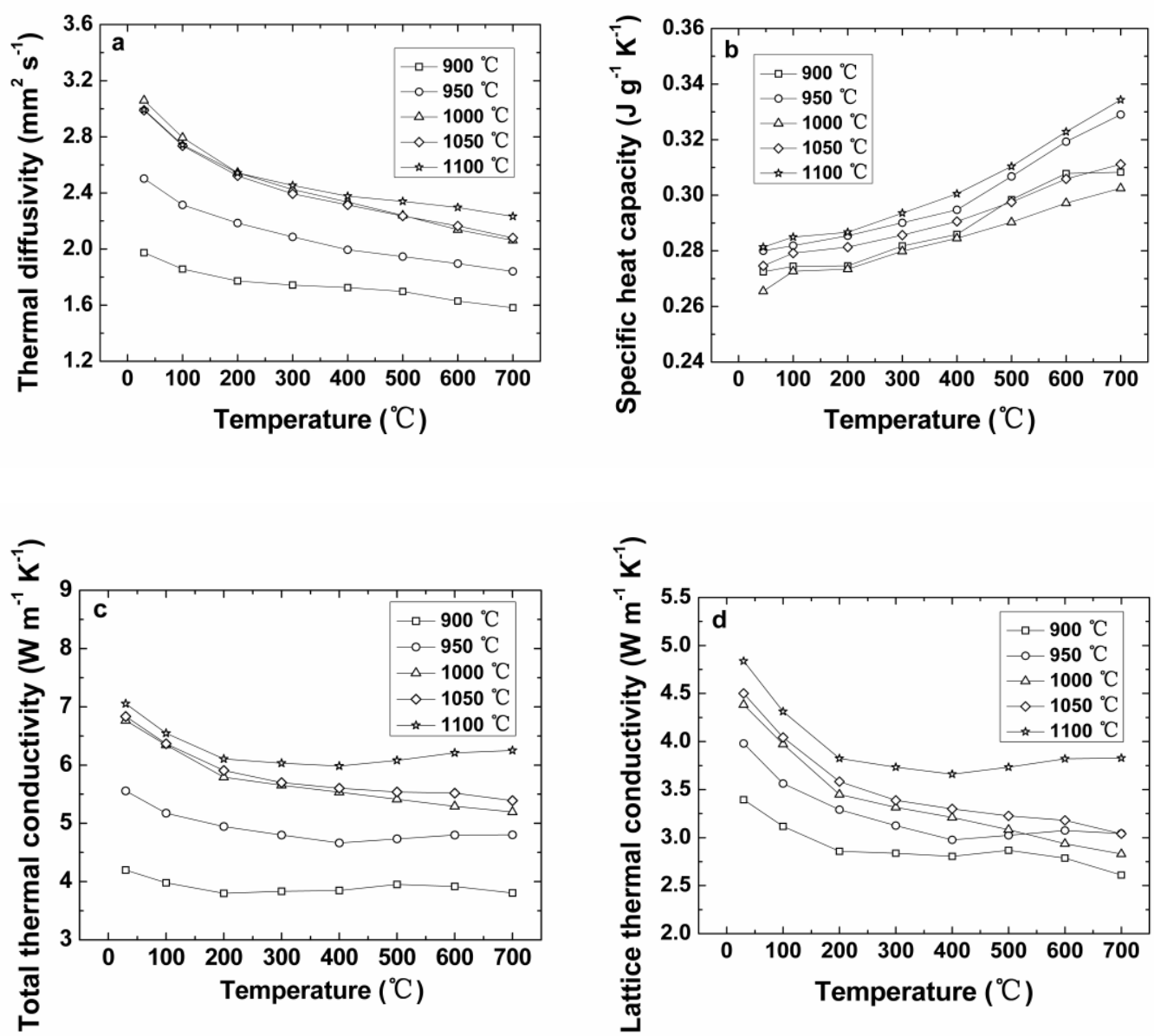

Figure 4 

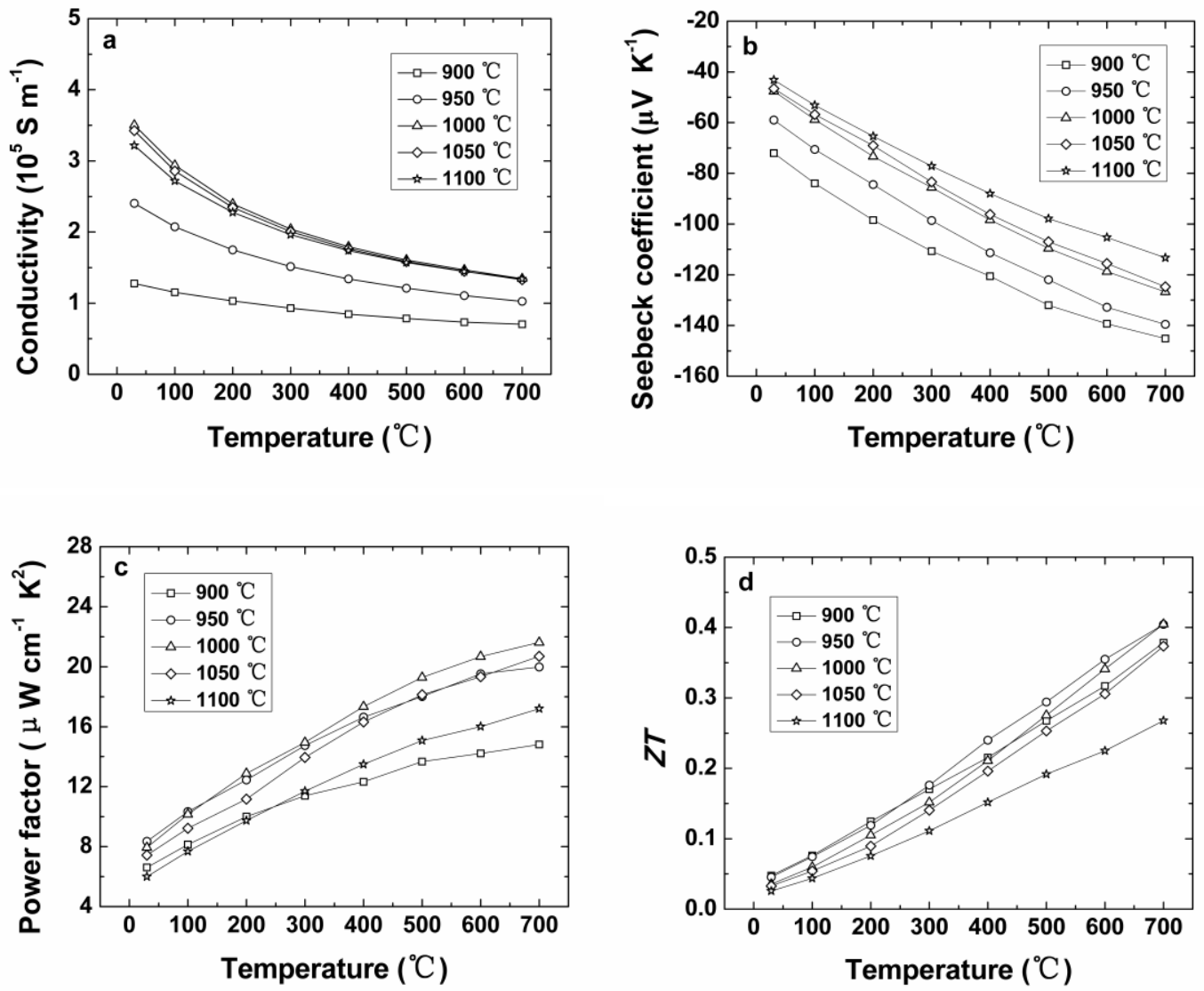

Figure 5 

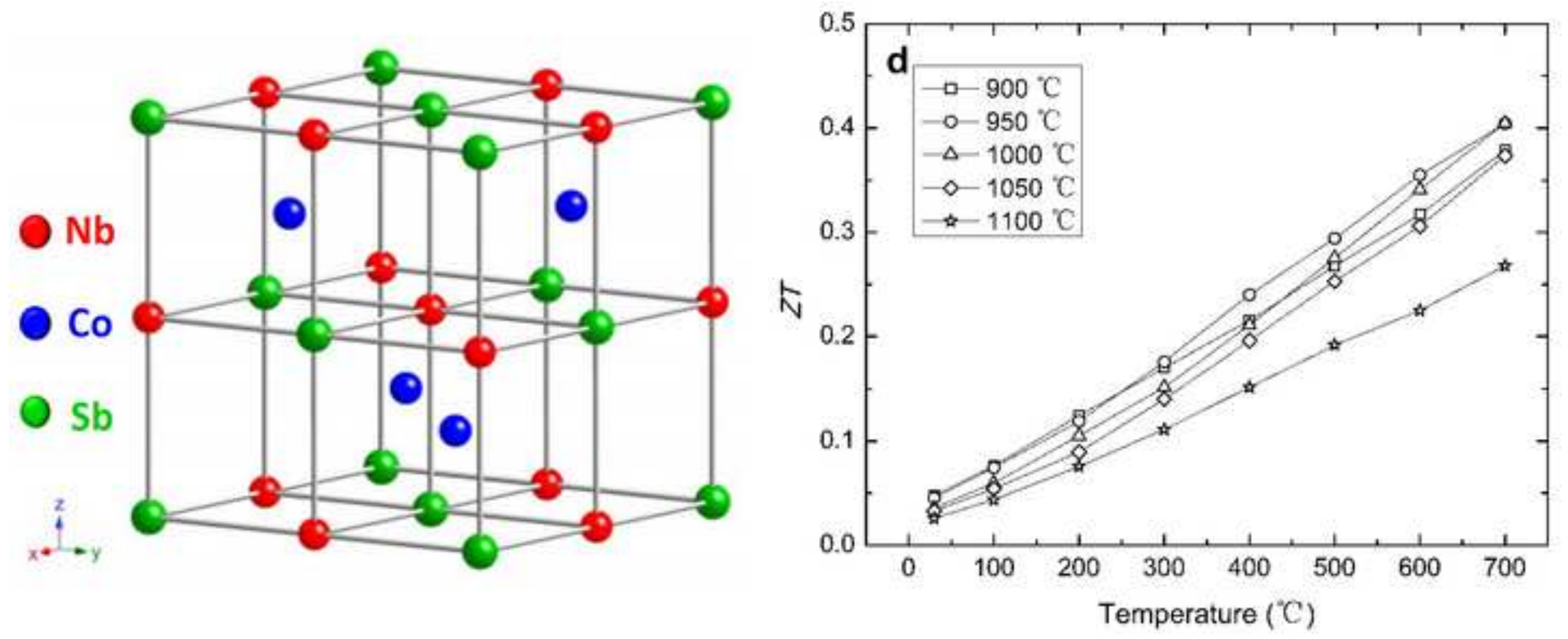